\title{
Promising agrofibers based on biodegradable polymers
}

\author{
Yulia Tertyshnaya ${ }^{1,2 *}$, Nelly Levina ${ }^{2}$, Maria Podzorova ${ }^{2}$ and Irina Bidey ${ }^{2}$ \\ ${ }^{1}$ Emanuel Instutute of Biochemical physics of RAS, 119991, Moscow, Russia \\ ${ }^{2}$ Federal Scientific Agroengineering Center VIM, 109428, Moscow, Russia
}

\begin{abstract}
Nonwoven fibrous materials from biodegradable polymers: polylactide and poly-3-hydroxybutyrate were obtained. Water vapor sorption by nonwoven fiber samples was investigated. It was determined that the substrate of non-woven material does not have a negative impact on the growth and development of cress plants. It is shown that the studied fiber samples are actively subjected to destruction in the soil, on the sample of PLA-PHB composition is decomposed faster than pure PLA.
\end{abstract}

\section{Introduction}

Currently, the agricultural sector seeks to use innovative agricultural technologies and materials that can increase and preserve the yield of different crops. It is assumed that the use of new materials from biodegradable polymers will increase productivity and improve product quality. Interest in natural polymers such as poly-3-hydroxybutyrate (PHB), polylactide (PLA), starch, chitin, cellulose, etc., increased in the past decade [1-3]. These polymers act either by absorbing the contaminants from any contaminated system (sorption mechanism) or by supplying carbon and energy to microorganisms to facilitate the denitrification mechanism. Biodegradation of polymers usually takes place in two main steps: primary degradation, in which fragmentation of the polymer chain occurs due to hydrolysis [4] or another oxidative reaction, and ultimate biodegradation, in which the microorganisms assimilate the low Mw chains formed.

PLA is a biodegradable thermoplastic polyester derived from biomass such as sugar, corn, beet, which possesses excellent physical and mechanical properties combined with biocompatibility and biodegradability properties [5]. Due to its initial production costs, the starting applications of PLA have been focused on high value products such as medical devices [6]. Because of the high market demand of biodegradable based products in recent years, PLA based products are manufactured commercially in many countries. Their applications include film, food packaging, textiles, disposal bottle and tableware [7].

PHB is the reverse polymer found in many types of bacteria, which can grow in a wide variety of natural environments, and can also be compostable. The main advantage of PHBs over other types of biodegradable polymers is its biodegradability under both aerobic and anaerobic conditions, such as buried in soil, immersed in activated sludge, and submerged

\footnotetext{
*Corresponding author: terj@rambler.ru
} 
in sea water. PHB is widely used for the development of bone implants, nerve fiber junctions, matrices for cell, filters and ultrafiltration membranes, not in agriculture for a while.

Polymer-based nanoscale composites can be produced by different fabrication methods. Electrospinning is one of the most prominently used techniques for producing micro- to nanometre fibers because it is a simple, cheap and versatile process. The electrospinning is based on a combination of mechanical and electrostatic forces applied to a solution or polymer melt orientated in an electric field. In particular, fibrous materials of PLA, PLA-PHB were prepared by electrospinning, having great potential for the agricultural applications.

\section{Experimental}

Preparation of Fibrous Material Poly(3-hydroxybutyrate) (Biomer, Germany) was used in the form of powder obtained by microbiological synthesis with a molecular mass of $2.5 \times 105$ $\mathrm{g} / \mathrm{mol}$, a melting point $(\mathrm{Tm})$ of $175-180^{\circ} \mathrm{C}$, and a density of $1.25 \mathrm{~g} / \mathrm{cm} 3$. To prepare the spinning solutions, chloroform of reagent grade was used. Polylactide (Nature works 4032D, USA) with a molecular mass of $1.7 \times 10^{5} \mathrm{~g} / \mathrm{mol}$ and a melting point (Tm) of $163-165^{\circ} \mathrm{C}$, and a density of $1.24 \mathrm{~g} / \mathrm{cm} 3$ was used. The fibers were obtained by electrospinning.

DSC The degree of crystallinity and melting temperature of the samples were studied with differential scanning calorimeter DSC 214 Polyma (Netzsch, Germany) at a heating rate of $10 \mathrm{~K} / \mathrm{min}$ and a sample weight of $5 \pm 0.1 \mathrm{mg}$. The temperature scale was calibrated against an indium standard $\left(\mathrm{Tm}=156.6^{\circ} \mathrm{C}, \Delta \mathrm{H}=28.44 \mathrm{~J} / \mathrm{g}\right)$.

The structure of the materials and its changes were studied by optical microscopy. The photomicrographs of the specimens were taken with an Axio Imager Z2m optical microscope (Carl Zeiss, Germany) using Axio Vision software at magnifi cations of 40, 100 and 200 in the transmitted and reflected light.

To study sorption, samples were taken of size $3 \times 3 \mathrm{~cm}$, pre-weighed on the electronic scales "Acculab ALC-110d4" (USA). Then all the samples were placed in an exicator, at the bottom of which was distilled water. The temperature of the experiment was $20=2{ }^{\circ} \mathrm{C}$. At certain intervals, the samples were weighed and, according to experimental data, sorption kinetics curves for both fiber samples were obtained.

Degradation in soil was investigated at $20=2{ }^{\circ} \mathrm{C}$. The microbial activity of soil was monitored with cotton along the extension of the experiment. The soil was maintained at approximately pH 7 and a relative humidity of $0.87 \mathrm{~g}$ water/g wet soil. Three specimens of each sample were extracted after certain times of burial in soil, cleaned and kept in a desiccator during 4 days in order to ensure water desorption before being analyzed.

\section{Results and discussion}

The obtained fibrous material can be used as a covering, for example, for germination of breeding seeds. Anyway, the material comes into contact with plants, soil, and it is important that the tested samples of nonwoven fiber from PLA and PLA-PHB do not have a negative impact on biological objects. The following experiment was carried out to evaluate the effect of nonwoven agrofiber on crops. Seeds of cress "Agur" was sown on a substrate of the agrofiber, which were in containers with soil. A control container with soil without the presence of agrofiber, in which were sown the seeds of cress. The number of seeds was the same everywhere. Fig. 1 shows the appearance of cress plants "Agur", grown on substrates of agrofibers and without them. It is noticeable that plants B1-B3 do not differ from the control. All plants look healthy and green. It was determined that the sowing qualities of cress seeds were higher by $2 \%$ in samples sprouted on nonwoven agrofiber. 


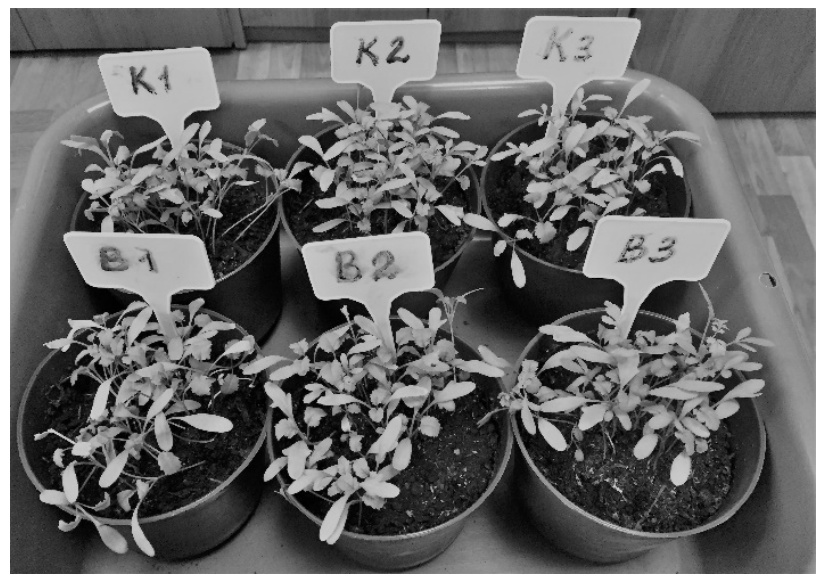

Fig. 1. View of cress lettuce (Lepidium sativum) grown in the traditional way (K1-K3) and on a polymer nonwoven fibers PLA-PHB (B1-B3).

Polymer materials used in agriculture are in contact with a wet environment or directly with water. It is very important to know the patterns of this interaction, so the kinetics of water vapor sorption has been studied. The results of the experiment on water vapor sorption by samples of nonwoven material are presented in Fig. 2. The kinetic sorption curves of both samples are identical. The relative mass increase $(\Delta \mathrm{m})$ of the PLA sample is very close to the PLA-PHB $\Delta \mathrm{m}$, thus both samples are capable of biodegradation by hydrolysis.

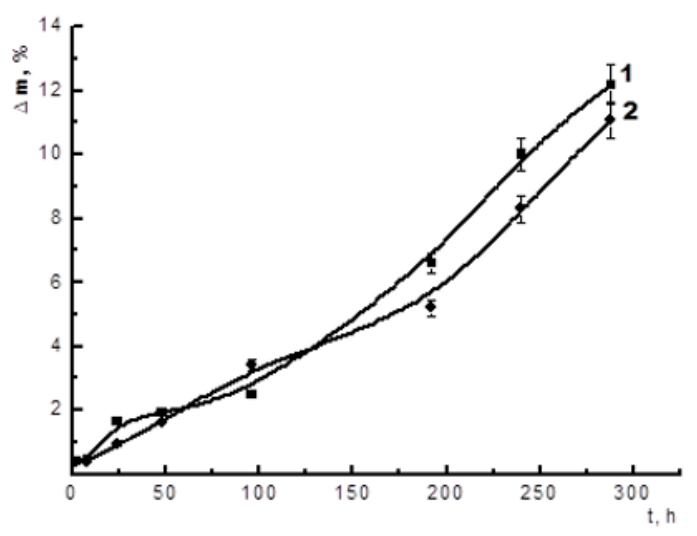

Fig. 2. Kinetics curves of distilled water vapor sorption by nonwoven samples from PLA (1) and PLA-PHB (2).

As mentioned earlier during the application of agrofiber inevitably gets into the soil, so the study of biodegradation in the soil of these materials is necessary. Biodegradation of polymers usually takes place in two main steps: primary degradation, in which fragmentation of the polymer chain occurs due to hydrolysis or another oxidative reaction, and ultimate biodegradation, in which the microorganisms assimilate the low Mw chains formed. Both the PLA fibrous material and the PLA-PHB sample should be well degraded in the soil due to the combined action of moisture (hydrolysis) and microorganisms (Fig. 3). 


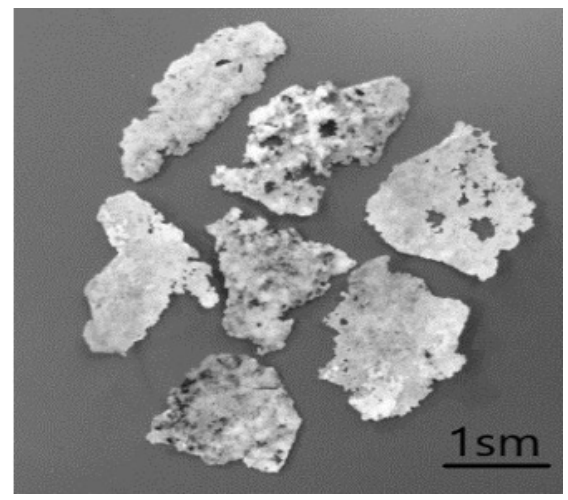

Fig. 3. Photographs of the PLA-PHB sample after 60 days degradation in soil.

Active biodestructors are fungi, which quickly affect polymeric materials based on natural polyesters. The most aggressive micromycetes in relation to PLA and PHB are Aspergillus Niger, Penicillium Chrysogenum and Trichoderma Viride. Microphotographs of samples of nonwovens from PLA and PLA-PHB obtained by electrospinning are shown in Fig.4.
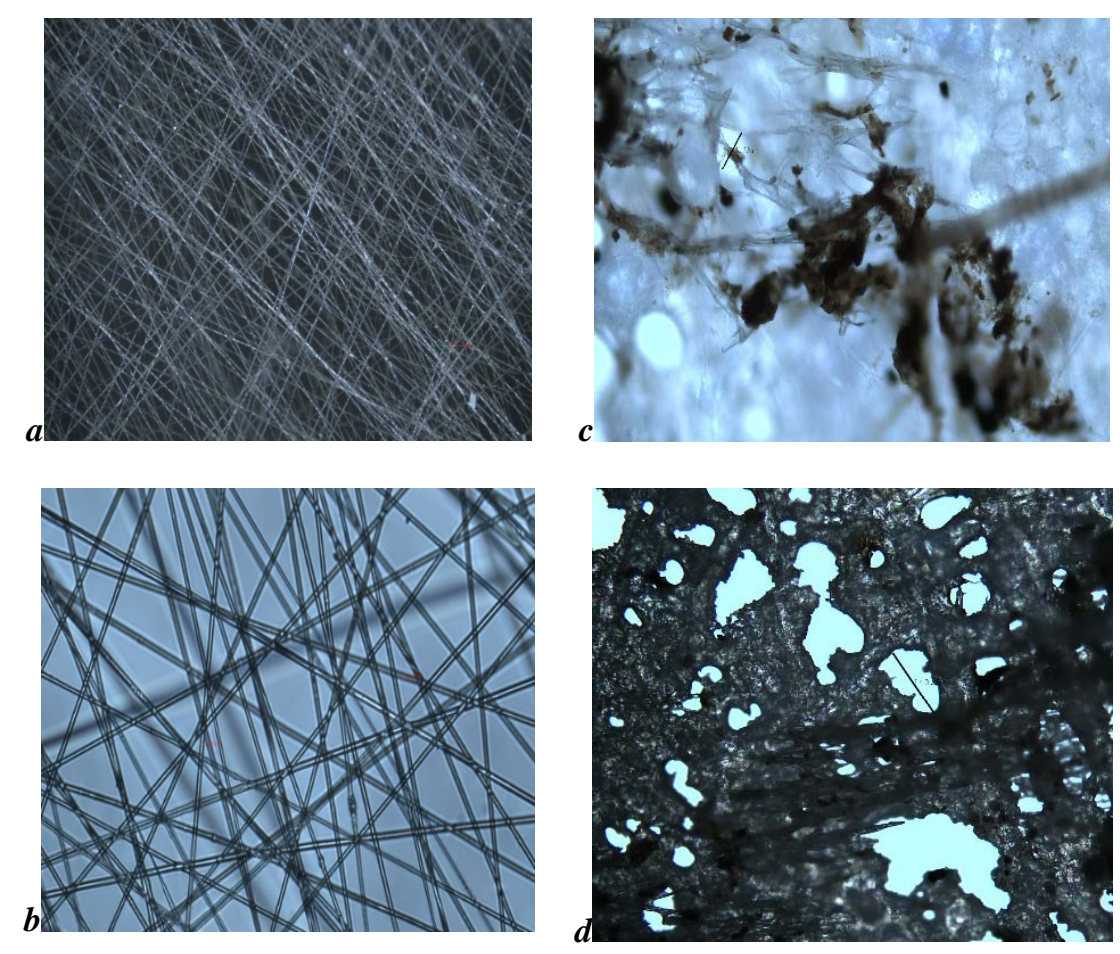

Fig. 4. Micrographs of the samples fibrous material: initial (a) - PLA and (b) - PLA-PHB, after degradation in soil during 60 days) - PLA and (d) - PLA-PHB.

One can see dirt and pores like the result of the degradation of the material. DSC data also confirm changes in the structure of the samples. The melting point decreases by $5-8{ }^{\circ} \mathrm{C}$ and the degree of crystallinity of both samples decreased. 


\section{Conclusion}

The investigated nonwoven fibrous materials do not have a negative effect on the morphology of plants and sowing qualities of seeds selected for the experiment.

It is shown that the obtained non-woven fibrous materials made of PLA and PLA-PHB well undergo hydrolytic degradation.

Nonwoven fiber samples are capable of biodegradation in the soil, which is confirmed by DSC microscopy.

In turn, the nature of the materials and their ability to biodegradation makes such materials promising for use in the agricultural sector.

\section{References}

1. Yu.V. Tertyshnaya, M.V. Podzorova, Rus. J. Appl. Chem. 91, pp. 417-423 (2018)

2. M.P. Arrieta, J. López, D. López, J.M. Kenny, L. Peponi, Ind. Crops. Prod. 93, pp. 290301 (2016)

3. Yu.V. Tertyshnaya, A.V. Khvatov, A.V. Lobanov, Rus. J. Phys. Chem B. 11, pp. 828832 (2017)

4. V. Speranza, A. De Meo, R. Pantani, Polym. Degrad. Stabl. 100, pp. 37-41 (2014)

5. E. T. H. Vink, K. R. Rábago, D. A. Glassner, P. R. Gruber, Polym. Degrad. Stab. 80, pp. 403-419 (2003)

6. R. Ortiz, S. Moreno - Flores, I. Quintana, MdM. Vivanco, J. R. Sarasua, J. L. Toca Herrera, Mater. Science and Engin.:C. 37, pp. 241-250 (2014)

7. D. Henton, P. Gruber, J. Lunt, J. Randall, Natur. fibres, biopolym. and biocomposites. 16, pp. 527-578 (2005) 